\title{
Dimer physics in the frustrated Cairo pentagonal antiferromagnet $\mathrm{Bi}_{2} \mathrm{Fe}_{4} \mathrm{O}_{9}$
}

\author{
K. Beauvois ${ }^{1 *}$, V. Simonet ${ }^{2}$, S. Petit ${ }^{3}$, J. Robert ${ }^{2}$, F. Bourdarot ${ }^{1}$, M.Gospodinov $^{4}$, A. A. Mukhin ${ }^{5}$, R. Ballou ${ }^{2}$, \\ V. Skumryev ${ }^{6} \&$ E. Ressouche ${ }^{1}$
}

\author{
${ }^{I} C E A$, IRIG/MEM-MDN, Grenoble, France, ${ }^{2}$ Institut Néel-CNRS, Grenoble, France, ${ }^{3}$ CEA-CNRS, LLB Saclay, France, ${ }^{4}$ Institute of \\ Solid State Physics, ${ }^{5}$ Bulgarian Academy of Sciences, Bulgaria, ${ }^{6}$ Russian Academy of Sciences, Russia, ${ }^{7}$ Universitat Autonoma de \\ Barcelona, Spain \\ *Current address: Institut Laue Langevin, Grenoble, France; beauvois@ill.fr
}

The research field of magnetic frustration is dominated by triangle-based lattices, but exotic phenomena can also be observed in pentagonal networks, as the well-known Cairo lattice. We demonstrate it here with the prototypical $\mathrm{Bi}_{2} \mathrm{Fe}_{4} \mathrm{O}_{9}$ material, where a peculiar noncollinear magnetic order is stabilized, resulting from the geometric frustration and a complex connectivity with three- and four-fold connected $\mathrm{Fe}_{1}$ and $\mathrm{Fe}_{2}$ sites (see Fig. 1) [1].

Firstly, we show the rich magnetic excitations, obtained by inelastic neutron scattering, where both collective excitations and local modes are present [2]. Those are related to local precession of pairs of spins due to the complex geometry of the lattice. From a comparison with spin wave calculations, we determined the antiferromagnetic superexchange interactions whose competition is confirmed to be at the origin of the spin arrangement. This analysis additionally unveils a hierarchy in the interactions, leading to a paramagnetic state constituted of strongly coupled dimers separated by much less correlated spins (see Fig. 1). This produces two types of response to an applied magnetic field associated with the two nonequivalent Fe sites, as observed in the magnetization distributions obtained using polarized neutrons.

In addition, we also report the preliminary results of the magnetic structure evolution of $\mathrm{Bi}_{2} \mathrm{Fe}_{4} \mathrm{O}_{9}$ under isostatic pressure (from 0 to $10 \mathrm{GPa}$ ), from powder neutron diffraction. At room temperature, we confirm the presence of a structural phase transition observed at approximatively 6.89(6) GPa by X-Ray diffraction and Raman spectroscopy [3]. From the low temperature patterns, we find a magnetic transition at this pressure toward a new magnetic arrangement that we discuss with respect to magnetic frustration.

Our study discloses, beyond the canonical examples of frustrated systems, novel behaviors that should be more generally observed in materials where the frustration is interlocked with complex connectivity and hierarchal interactions.

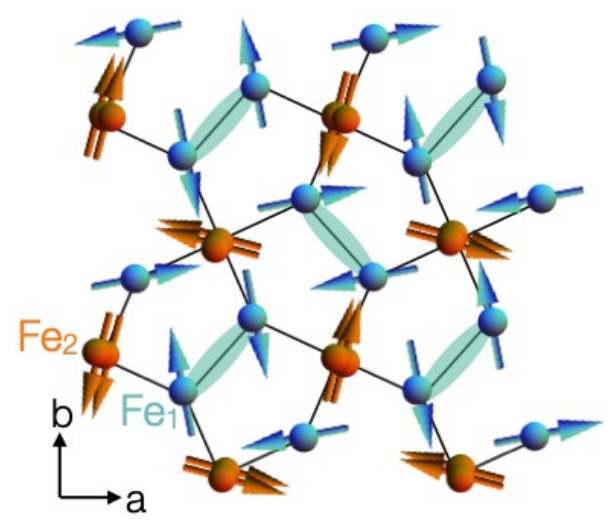

Figure 1. Magnetic arrangement of the $\mathrm{Fe}_{1}$ (in blue) and $\mathrm{Fe}_{2}$ (in orange) on the pentagonal lattice. Blue ellipses underline strongly coupled antiferromagnetic $\mathrm{Fe}_{1}$ spins.

[1] Ressouche, E., Simonet, V., Canals, B., Gospodinov, M. \& Skumryev, V. (2009). Phys. Rev. Lett. 103, 267204.

[2] Beauvois, K., Simonet, V., Petit, S., Robert, J., Bourdarot, F., et al, (2020). Phys. Rev. Lett. 124, 127202.

[3] Friedrich, A., Biehler, J., Morgenroth, W., Wiehl, L., Winkler, B., et al, (2012). Journal of Physics: Condensed Matter $24,145401$.

Keywords: magnetic frustration; pentagonal lattice; magnetic interactions; spin dynamics; neutron scattering 